\title{
Effects of perceptual similarity but not semantic association on false recognition in aging
}

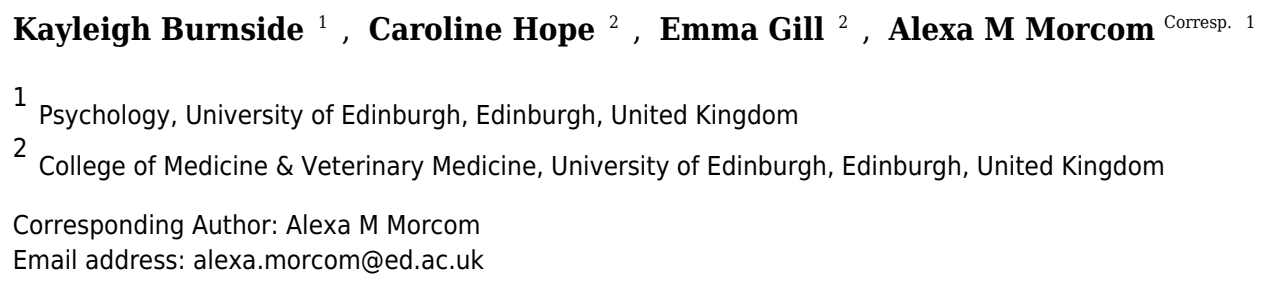

This study investigated semantic and perceptual influences on false recognition in older and young adults in a variant on the Deese-Roediger-McDermott paradigm. In two experiments, participants encoded intermixed sets of semantically associated words, and sets of unrelated words. Each set was presented in a shared distinctive font. Older adults were no more likely to falsely recognize semantically associated lure words compared to unrelated lures also presented in studied fonts. However, they showed an increase in false recognition of lures which were related to studied items only by a shared font. This increased false recognition was associated with recollective experience. The data show that older adults do not always rely more on prior knowledge in episodic memory tasks. They converge with other findings suggesting that older adults may also be more prone to perceptually-driven errors. 
Effects of perceptual similarity but not semantic association on false recognition in aging. Kayleigh Burnside ${ }^{1}$, Caroline Hope ${ }^{2}$, Emma Gill ${ }^{2}$ and Alexa M. Morcom ${ }^{1}$

${ }^{1}$ Psychology, University of Edinburgh.

${ }^{2}$ College of Medicine and Veterinary Medicine, University of Edinburgh.

Author Note

The first two authors contributed equally to the paper. Correspondence regarding this article should be addressed to Alexa M. Morcom, Psychology, University of Edinburgh, 7 George Square, Edinburgh EH8 9JZ, United Kingdom. Email: alexa.morcom@ed.ac.uk 


\begin{abstract}
This study investigated semantic and perceptual influences on false recognition in older and young adults in a variant on the Deese-Roediger-McDermott paradigm. In two experiments, participants encoded intermixed sets of semantically associated words, and sets of unrelated words. Each set was presented in a shared distinctive font. Older adults were no more likely to falsely recognize semantically associated lure words compared to unrelated lures also presented in studied fonts. However, they showed an increase in false recognition of lures which were related to studied items only by a shared font. This increased false recognition was associated with recollective experience. The data show that older adults do not always rely more on prior knowledge in episodic memory tasks. They converge with other findings suggesting that older adults may also be more prone to perceptually-driven errors.
\end{abstract}

\title{
Abbreviations
}

DRM, M, SD, ANOVA, MSE, BF 
It is well established that memory for events is impaired in even healthy aging (Light,

4 1991). An emerging hypothesis is that prior knowledge, which is well maintained in older age,

5 can support declining episodic memory to some degree (Naveh-Benjamin et al., 2003; Castel,

6 2005; Umanath \& Marsh, 2014). However, this support may also be a "double-edged sword"

7 (Reder, Paynter, Diana, Ngiam, \& Dickison, 2007), bringing a greater cost in memory errors than

8 in the young. Consistent this view, older adults appear to be particularly susceptible to semantic

9 as opposed to perceptual influences on false memory for categorically related similar pictures

10 (Koutstaal et al., 2003; Pidgeon \& Morcom, 2014). To investigate whether the same pattern

11 would be found in a verbal false memory task, we contrasted the effects of associative and

12 perceptual relatedness on false recognition in older and young adults in two experiments.

Although older adults are widely regarded as more prone to false memory (e.g., (Devitt \&

14 Schacter, 2016; Mitchell \& Johnson, 2009; Schacter, Koutstaal, \& Norman, 1997), the reality

15 may be more nuanced. In the categorized pictures paradigm, older adults are more likely to

16 falsely recognize pictures of objects they have not studied when the lures are related to studied

17 items by membership of the same basic-level category (e.g., a picture of a different dog;

18 Koutstaal \& Schacter, 1997; Koutstaal, Schacter, Galluccio, \& Stofer, 1999; Lovden, 2003).

19 These errors are attributed to a greater reliance on processing semantic gist compared to specific

20 item and contextual information (Koutstaal \& Schacter, 1997). Older adults are also often

21 reported to show increased false recognition when lures are associatively rather than

22 categorically related to studied items, in the Deese-Roediger-McDermott paradigm (Deese, 1959;

23 Roediger \& McDermott, 1995; Intons-Peterson, Rocchi, West, McLellan, \& Hackney, 1999;

24 Norman \& Schacter, 1997; Schacter, Israel, \& Racine, 1999; see also Rankin \& Kausler, 1979), 
25 although more consistently so when false memory is tested by recall than by recognition (see

26 Gallo, 2006, pp. 184-202 for review). This, too, has been attributed to an increase in gist-based

27 memory (Tun, Wingfield, Rosen, \& Blanchard, 1998; Brainerd \& Reyna, 2002)

28 There have been few direct investigations of the specific roles that semantic and

29 perceptual information play in these memory errors, as lures often share both types of relations

30 with studied items. Koutstaal et al. (2003) found heightened false recognition in older adults for

31 pictures of unstudied objects sharing basic-level category membership with studied items (e.g.

32 candles), but not for items from visually similar 'abstract' novel object sets. A similar effect was

33 observed when novel objects were given verbal labels, leading the authors to propose that older

34 adults rely more on overt verbal semantic categorization than the young. We recently replicated

35 Koutstaal et al.'s (2003, Experiment 2) finding of a stronger age-related increase in false

36 recognition for pre-experimentally meaningful picture lures compared to lures which were

37 members of novel, 'abstract' categories, consistent with specific importance of semantic

38 relatedness. However, unlike Koutstaal et al., we also found an age-related increase in errors for

39 the 'abstract', visually similar lures (Pidgeon \& Morcom, 2014). It is possible that the latter was

40 due to greater incidental semantic processing of the multi-item 'abstract' categories by older

41 adults. However, converging evidence that their greater susceptibility to false recognition is not

42 driven entirely by semantic processing comes from studies finding age-related increases in false

43 recognition of phonologically similar as well as semantically associated lures (Budson, Sullivan,

44 Daffner, \& Schacter, 2003; Sommers \& Huff, 2003; Watson, Balota, \& Sergent-Marshall, 2001;

45 see also Rankin \& Kausler, 1979). To our knowledge, no study has specifically examined visual

46 perceptual influences on false recognition in older adults. Thus, the nature of perceptual 
47 influences on false recognition in aging is currently unclear, as are the conditions under which

48 older adults rely more on semantic information than the young.

The current study examined older and young adults' susceptibility to semantic and

50 perceptual influences on false recognition using a variant on the DRM paradigm (Figure 1). The

51 manipulations of associative and perceptual relatedness using semantically associated words in

52 fancy fonts were taken from Arndt and Reder's (2003) study in young adults. Experiment 1's

53 design was otherwise based on Koutstaal et al.'s study (2003; Experiment 2) and our subsequent

54 replication and extension (Pidgeon \& Morcom, 2014). This meant that study and test lists were

55 intermixed rather than blocked, as is usual in the categorized pictures paradigm, unlike in the

56 DRM paradigm, in which blocked (list-wise) presentation is typical. In the Correlated Font

57 conditions, sets of associated DRM words were studied in the same font. At test, studied items

58 and critical and related lures were also presented in this font. In the Font Only conditions, sets of

59 unrelated items were studied in the same font. At test, studied items and lures were also

60 presented in this font. Experiment 2 included an additional condition aimed at assessing

61 interactions between font and semantic information.

62 Based on our earlier findings in the categorized pictures task, we expected that older

63 adults would show an increase in false recognition for semantically-related than perceptually-

64 related lures, relative to the young, and a (smaller) increase in false recognition of perceptually-

65 related lures (Koutstaal et al., 2003; Pidgeon \& Morcom, 2014). We were also interested in

66 whether age-related differences in memory errors due to semantic and perceptual relations were

67 accompanied by a subjective experience of recollection. We measured the quality of recognition

68 using the Remember-Know (RK) procedure (Mandler, 1980). This helps to disambiguate false

69 memory from age-related differences in responses based directly on semantic knowledge or 
70 guessing. Previous work suggests that age-related increases in false recognition are partly driven

71 by an increase in illusory recollection (McCabe, Roediger, McDaniel, \& Balota, 2009).

72 However, findings from DRM tasks have been mixed: Intons-Peterson et al. (1999) showed

73 increased false recollection in older adults using the RK procedure, as did Gallo et al. (2003)

74 using a source memory task, but two other studies using RK did not (Schacter, Israel and Racine,

75 1999; Skinner \& Fernandes, 2009).

76

77

78

79

80

81

82

83

84

85

86

87

\section{Experiment 1}

\section{Method}

Participants

Participants were 24 young (19-23 years, $M=20.4)$ and 24 older adults $(61-73$ years, $M$ $=66.5)$. We previously estimated that group sizes of 24 were necessary to replicate Koutstaal et al.'s (2003) critical Stimulus Type by Age interaction with .95 power (effect size $\mathrm{f}=.513$; see Pidgeon $\&$ Morcom, 2014). This $\mathrm{N}$ would also yield power $=.78$ to detect an age-related increase in false recollection due to perceptual similarity (effect size $d=.704$ from Pidgeon $\&$ Morcom, 2014; one-tailed test). All participants were native English speakers with normal or corrected-to-normal vision. Baseline cognitive tests showed higher crystallized IQ in the older group than in the young, as expected (Wechsler Test of Adult Reading standardized score in young $\mathrm{M}=116$, older $\mathrm{M}=123, \mathrm{SD}=6.5$ and 5.0; $t(42.2)=4.15, \mathrm{p}<.001, \mathrm{~d}=1.2$; Holdnack, 2001) and no difference in forward Digit $\operatorname{Span}(\mathrm{M}=7.3$ and $7.5, \mathrm{SD}=1.1$ and $1.3 ; t(45.6)=.46$, $\mathrm{p}=.650$, with moderate Bayesian evidence for the null hypothesis, $\mathrm{BF}_{10}=3.3$, range $=2.6$ for prior width .5 to 4.0 for width .9). The older group showed a typical decrement on Digit Symbol

1 Coding (Wechsler Adult Intelligence Scale IV; Wechsler, 2008; young $M=71.3$, old = 56.9, SD $=12.3$ and $10.3 ; t(44.7)=4.42, \mathrm{p}<.001, \mathrm{~d}=1.3)$ and were slower on part B relative to part A of 
93 the Trail Making test (Reitan, 1958; for difference, $t(32.8)=4.0, \mathrm{p}<.001, \mathrm{~d}=1.2$; consistent

94 with reduced executive function and processing speed (Salthouse, Atkinson \& Berish, 2003).

95 Written consent was obtained from all participants. The study was approved by the Psychology

96 Research Ethics Committee, University of Edinburgh (ref. 72-1314/2).

97 Materials

98 Stimuli were constructed using words written in distinctive fonts. The three experimental 99 conditions are illustrated in Figure 1. In the Correlated Font conditions, sets of associated words

100 from DRM lists were studied in the same font, and lures were related to studied items by both

101 semantic association and font. Two types of lure were included at test, making two Correlated

102 Font conditions: those with DRM critical associates as lures (Correlated Font-Critical), and those

103 with unstudied associates from the studied list as lures (Correlated Font-Related). In the Font

104 Only condition, sets of unrelated words were studied in the same font, and additional unrelated

105 words served as lures and were presented in the same font at test.

106

$<$ Figure 1 about here $>$

107 We used 512 words and 80 distinctive fonts (from www.urbanfonts.com and

108 http://www.1001 freefonts.com/). Thirty two 11-word associated DRM lists were drawn from the

109 University of South Florida (USF) Free Association Norms database

110 (http://www.usf.edu/FreeAssociation/; (Nelson, McEvoy, \& Schreiber, 2004), selected to

111 maximize Backward Associative Strength (BAS) from list items to their Critical lures (mean =

112 .48). Mean BAS for list words to other list words used as Related lures was .0065. Sets of 11

113 unrelated words were also constructed using words from the MRC Psycholinguistic database

114 (Wilson, 1988), selected to match the associated DRM words on word length and frequency

115 (median length $=6$ and written frequency $=32$ ). Each study phase list comprised 432 items, 
116 including 9 words from each of 16 associated lists per DRM condition (16 lists for Correlated

117 Font-Critical and 16 for Correlated Font-Related), and 9 words from each of 16 unrelated sets of

118 words for the Font Only condition. Each test phase list comprised 128 items, and included 1

119 studied word and one lure for each studied list (16 studied items and 16 lures per condition). Test

120 lists also contained 32 Novel words. Sixteen of the Novel words were separately taken from the

121 MRC database to match the Critical lures on word length and frequency (Critical-matched;

122 median length $=5$ and written frequency $=110$ per million). The other 16 Novel words were

123 taken from the unrelated word sets, and therefore matched to the studied associated items, related

124 lures in the Correlated Font-Related condition, and unrelated words in the Font Only condition.

125 To counterbalance the stimuli over participants, we randomly allocated fonts and words to

126 conditions several times, and randomly allocated the counterbalanced lists to participant

127 numbers. First, DRM list words were allocated to serve as studied items for the Critical and

128 Related lure conditions, and Related lures. Next, unrelated words were allocated to serve as

129 studied (Font Only) or Novel items. Fonts were then allocated to the associated and unrelated

130 sets of words, and to studied and Novel items. Young and older groups received precisely the

131 same lists.

132 Procedure

133 Participants completed a single study-test cycle after a short practice. They viewed single

134 words in distinctive fonts at the center of a computer screen. At study, they completed an

135 incidental encoding task, judging the degree to which the word's font fit its meaning with a 4-

136 way button press response (Very Well to Very Poorly). At test, participants were asked to

137 "decide which words are new and which are old and what, if anything you remember about the

138 words you have seen before", with no instruction regarding font. We used a variant of the 
139 Remember/Know procedure with standard instructions (Gardiner, 1988; Tulving, 1985), though

140 we replaced the term 'Know' with 'Familiar' for clarity (see Migo, Mayes \& Montaldi, 2012).

141 Participants judged whether they recollected seeing a word ('Remember'; R), or whether it was

142 just familiar ('Familiar'; F), or new ('New'), or they could not decide whether it was studied

143 ('Guess'). At study and test, items remained on-screen until a response was made for up to 7000

144 msec, followed by 1500 msec fixation.

145 Data analysis

146 All degrees of freedom and p values were Greenhouse-Geisser corrected, and Welch's

147 unequal variances $t$-test was used where appropriate. We also supplemented null-hypothesis

148 significance testing with Bayes Factor (BF) analyses using JASP (https://jasp-stats.org/; version

149 0.8.0.1; Rouder, Speckman, Sun, Morey, \& Iverson, 2009). Bayes Factors were used to provide

150 evidence for the null hypothesis where this was important for interpretation of findings, and for

151 summary analyses across experiments. Although categorization of levels of Bayesian evidence is

152 necessarily arbitrary, for clarity we adopt the labels used in JASP (Lee \& Wagenmakers, 2013),

153 i.e., that $\mathrm{BF}<3$ indicates "anecdotal" evidence, $3<\mathrm{BF}<10$ "moderate", $10<\mathrm{BF}<30$

154 "strong", $30<\mathrm{BF}<100$ "very strong", and BF $>100$ "extreme" evidence). The Bayesian $t$-tests

155 used uninformative Cauchly priors with $\mathrm{M}=0$, width $=.71$, with additional robustness checks of

156 BFs under a range of prior distribution widths (width $=.5$ or .9 ) to check that this did not

157 substantially change the results (a wider prior increases evidence for the null hypothesis).

\section{Results}

159 Table 1 summarizes the overall response proportions (collapsed over $\mathrm{R}$ and F), and

160 Figure 2 (A-B) illustrates true and false recollection. To assess true and false recognition we

161 analysed both raw response proportions and proportions of hits (correctly identified studied 
162 items) and false alarms (FA) after adjusting for FA to novel items (i.e, subtracting novel FA

163 proportions; see e.g., Koutstaal et al., 2003). For hits, the corrected measure was equivalent to

164 the $P_{r}$ discrimination index which assumes two-high-threshold dual process recognition

165 (Snodgrass \& Corwin 1988). Hit proportions for Correlated Font items were collapsed over the

166 Critical and List conditions (as the studied items were equivalent). For Critical lures, the

167 corresponding Novel items were Critical-Matched, and for studied items and for List and Font

168 Only lures they were Related-matched (see Materials). Lastly, we assessed recollection, using

169 the proportion of hits and lure false alarms which attracted "Remember" (R) responses (Figure 2, 170 A-B).

$<$ Table 1 about here $>$

172 True recognition.

173 True recognition was better in both age groups for items in the Correlated Font than the

174 Font Only condition, and better on one of the measures in the young than the older group.

175 ANOVA on raw hits with factors of Condition (Correlated Font/ Font Only) and Group (Young/

176 Older) showed a main effect of Condition, $F(1,46)=17.10, \mathrm{MSE}=.18, \mathrm{p}<.001 ; \eta^{2} \mathrm{p}=.27$; see

177 Table 1 and Fig 2. For raw proportions of hits to studied items it was unclear whether or not the

178 groups differed: for main effect of Group, $F(1,46)=.99, \mathrm{MSE}=.06, \mathrm{p}=.325, \mathrm{BF}_{01}$ for the null

179 hypothesis $=1.9$; for Condition $\mathrm{x}$ Group, $F(1,46)=.02$, MSE $<.001, \mathrm{p}=.89$. Discrimination of

180 studied from new items $\left(P_{r}\right)$ was also greater in the Correlated Font condition, $F(1,46)=18.23$, $\mathrm{p}$

$181<.001, \eta_{\mathrm{p}}^{2}=.28, \mathrm{MSE}=.19$, and unlike for raw recognition this adjusted measure showed

182 greater true recognition in the young group, $F(1,46)=6.48, \mathrm{MSE}=.58, \mathrm{p}=.014, \eta_{\mathrm{p}}^{2}=.12$. The

183 interaction was not significant, $F(1,46)=.02, \mathrm{MSE}<.001, \mathrm{p}=.90$, although there was no

184 Bayesian evidence against one, $\mathrm{BF}_{01}=.95$. For true recollection, the Correlated Font and Font 
185 Only conditions again differed (for main effect, $F(1,46)=23.4$, MSE $=.27, \mathrm{p}<.001, \eta_{\mathrm{p}}^{2}=.34$,

186 but age effects for this measure were unclear: for main effect, $F(1,46)=1.22, \mathrm{MSE}=.01, \mathrm{p}=$

187.28 , with anecdotal Bayesian evidence for the null, $\mathrm{BF}_{01}=1.3$; for Condition $\mathrm{x}$ Group, $F(1,46)=$

$1881.7, \mathrm{MSE}=.17, \mathrm{p}=.19$. The presence of only minor age differences in true recognition meant

189 that effects on false recognition were more straightforward to interpret.

190

$<$ Figure 2 about here $>$

191 False recognition.

192 For Novel items, ANOVA on proportions falsely recognized with factors of Condition

193 (Critical-Matched/ Related-matched) and Group (Young/ Older) showed a slightly higher

194 baseline false alarm rate in the older group, reflected in a main effect of Group, $F(1,46)=4.20, \mathrm{p}$

$195=.047 ; \eta_{\mathrm{p}}^{2}=.08, \mathrm{MSE}=.32$. The conditions did not differ significantly, for main effect of

196 Condition, $F(1,46)<.001, \mathrm{p}=1.00$, nor did the interaction, $F(1,46)=.60, \mathrm{p}=.442$, MSE $=.002$,

197 although Bayesian evidence against the latter effect was only anecdotal, with $\mathrm{BF}_{01}$ for the null

198 hypothesis $=2.3$. No condition or group effects were significant for proportions of falsely

199 recollected novel items either, and there was strong Bayesian evidence against an interaction (for

200 main effect, $F(1,46)=2.43, \mathrm{MSE}=.002, \mathrm{p}=.126, \mathrm{BF}$ against inclusion in model $=3.7$; for

201 interaction, $F(1,46)=.09, \mathrm{MSE}=.00007, \mathrm{p}=.768, \mathrm{BF}$ against inclusion $=55.6)$. The presence

202 of group differences in raw Novel FA suggests caution may be needed in interpretation of Novel-

203 adjusted false recognition measures, even though this effect did not differ by condition.

204 Across the two groups, lure false recognition was highest for Correlated Font Critical

205 lures, intermediate for Correlated Font Related lures and lowest for Font Only lures (Table 1 and

206 Fig 2). The effects of age Group also depended on Condition for both raw and adjusted false

207 recognition. ANOVA on raw lure FA with factors of Condition (Critical lure/ Related lure/ Font 
208 Only lure) and Group (Young/ Older) revealed a main effect of Condition, $F(1.7,77.6)=63.1$, $209 \mathrm{MSE}=.23, \mathrm{p}<.001, \eta_{p}^{2}=.53$, and interaction with Group, $F(1.7,77.6)=9.97, \mathrm{p}<.001, \eta_{\mathrm{p}}^{2}=$ $210.18, \mathrm{MSE}=.23$; for main effect of Group, $F(1,46)=1.5, \mathrm{MSE}=.19, \mathrm{p}=.23$. Post hoc tests

211 showed a significant increase in Font Only lure false recognition in the older group compared to 212 the young, $t(30.6)=-2.88, \mathrm{p}=.007, \mathrm{~d}=.83$; alpha $=.017$. In the Correlated Font conditions, age 213 effects on false recognition were non-significant for both Critical and Related lures, although 214 Bayes Factors showed only anecdotal evidence for the null hypothesis, $\mathrm{t}(41.2)=.89, \mathrm{p}=.38$, $215 \mathrm{BF}_{01}=2.5 ; \mathrm{t}(36.8)=-1.65, \mathrm{p}=.108 ; \mathrm{BF}_{01}=1.2$. These findings suggested that older adults 216 showed an increase only in perceptually-driven false recognition, but we also analyzed false 217 recollection and adjusted measures to rule out effects of response criterion.

218 For false recollection ANOVA with the same factors again showed an interaction of 219 Condition $x$ Group, $F(1.7,80.4)=6.0, \mathrm{MSE}=.088, \mathrm{p}=.005, \eta_{p}^{2}=.12$, as well as a main effect 220 of Condition, $F(1.7,80.4)=30.0, \mathrm{MSE}=.44, \mathrm{p}<.001, \eta_{p}^{2}=.37$, but not Group, $F(1,46)=1.0$,

$221 \mathrm{MSE}=.12, \mathrm{p}=.32$. Post hoc tests did not show a significant age-related increase in any 222 condition (for Font Only lures, $t(24.3)=-2.31, \mathrm{p}=.030$; for Critical lures, $t(40.6)=.40, \mathrm{p}=.695$, 223 for Related lures, $t(31.3)=1.13, \mathrm{p}=.268$; alpha $=.017$ ). Bayes factors showed anecdotal 224 evidence for the alternative hypothesis in the Font Only condition, $\mathrm{BF}_{10}=2.4$, (range $=2.5$ for 225 prior width .5 to 2.2 for width .9), and moderate Bayesian evidence for the null hypothesis for 226 the Critical lures, $t(40.1)=.40, \mathrm{p}=.70, \mathrm{BF}_{01}=3.3($ range $=2.5$ to 4.0$)$, but anecdotal evidence 227 for the Related lures, $t(31.3)=-1.12, \mathrm{p}=.27, \mathrm{BF}_{01}=2.1$. Although this pattern qualitatively 228 supported the results for raw FA, there was not enough evidence to clearly determine whether the 229 increase in false recognition of Font Only lures by older compared to young adults was 230 accompanied by an increase in false recollection. 
232 recognition (Table 1). ANOVA again showed a main effect of Condition, $F(1.8,82.2)=61.35, \mathrm{p}$

$233<.001, \eta_{\mathrm{p}}^{2}=.57, \mathrm{MSE}=1.43$, and an interaction of Condition $\mathrm{x}$ Group, $F(1.8,82.2)=12.00, \mathrm{p}<$

$234.001, \eta_{\mathrm{p}}^{2}=.21, \mathrm{MSE}=.28$; for main effect of Group, $F(1,46)=1.2, \mathrm{MSE}=.04, \mathrm{p}=.28$, but post

235 hoc tests showed that this reflected group differences for Critical rather than Font Only lures.

236 While older adults falsely recognized a similar (though numerically larger) number of Font Only

237 Lures to the young, $\mathrm{t}(46)=1.69, \mathrm{p}=.097, \mathrm{BF}_{01}$ for the null hypothesis $=1.0$, on this measure

238 they falsely recognized significantly fewer Critical Lures, $\mathrm{t}(46)=3.03, \mathrm{~d}=.78, \mathrm{p}=.004, \mathrm{BF}_{10}$ for

239 the alternative hypothesis $=6.8$, range $=6.7$ for prior width $=.5$ to 6.6 for width $=.9$; for Related

240 lures, $\mathrm{t}(46)=.07, \mathrm{p}=.944, \mathrm{BF}_{01}=3.4$, range $=2.7$ to $4.2 ;$ alpha $=.017$. This apparent age-related

241 reduction in adjusted false recognition had not been predicted.

242 Discussion

243 The results show an age-related increase in perceptual but not associative false

244 recognition in this task. Counter to our prediction, age effects on false recognition and

245 recollection were less prominent for the semantically and perceptually related Correlated Font

246 Critical lures than for the perceptually-related Font Only lures. However, because of the different

247 finding for the corrected FA measure, it is not possible to determine unambiguously which

248 condition carried the age effect.

While numerous previous studies have reported age-related increases in associative false recognition, others have also found null effects (e.g. Kensinger \& Schacter, 1999; Gallo \&

251 Roediger, 2003; Kensinger \& Schacter, 1999; Intons-Peterson, Rocchi, West, McLellan, \&

252 Hackney, 1999; Skinner \& Fernandes, 2009; see Gallo 2006, pp. 193-189). Some of the

253 differences between studies, and the present discrepancy between raw and adjusted false 
254 recognition findings, probably reflect differences in the selection of items for baseline (novel)

255 conditions. It is important to demonstrate that age-related differences in false recognition reflect

256 differences in memory rather than in decision criterion (see Roediger, Watson, McDermott, \&

257 Gallo, 2001). One way to do this is to adjust for baseline FA to novel items (e.g., Koutstaal et al.,

258 2003). It is common for DRM studies to counterbalance stimuli so that list items and critical

259 lures from unstudied lists serve as Novel items. However, the Novel items are typically either

260 DRM list items from different list positions from those studied, or a mix of unstudied list items

261 and unstudied critical lures (examples of exceptions: McCabe \& Smith, 2002; Schacter, Israel, \&

262 Racine, 1999). Therefore, adjusted critical lure false recognition may often not fully correct for

263 baseline age-related differences in responding, because of differences between critical lures and

264 novel items on indices such as familiarity and imageability. In the present study we did not

265 counterbalance Novel items and lures but did match them on word length and frequency. Thus,

266 the apparent age-related reduction in adjusted Critical lure false alarms was likely influenced by

267 differences in young and older adults' responses due to unmeasured item characteristics between

268 the Critical Lure and Critical-Matched Novel conditions. More importantly, it was possible that

269 the finding of a differential age effect on associative and perceptually-driven false recognition

270 reflected subtle differences in the two groups' false recognition of Critical-Matched and Related-

271 Matched Novel items. There was no clear evidence for this, as age effects on Novel false alarms

272 were equivalent in the two conditions. However, replication in a study with fully

273 counterbalanced and matched Novel items was essential.

274 We also wanted to test a possible mechanism of an age-related reduction in associative

275 false recognition in this task, if present. Two known modifiers of age-related differences in false

276 memory are individual differences in prefrontal cortex function and the post-retrieval monitoring 
277 demands of the task (Gallo, 2006). These are unlikely to account for the current data. The older

278 group had high verbal IQ, but still showed typical reductions on measures of processing speed

279 and executive function. Thus, they were unlikely to be better than the young at retrieval

280 monitoring. However, reduced activation at encoding was a possibility. Lure activation is

281 thought to be automatic and preserved in old age (Gallo \& Roediger, 2003; Koutstaal, 2003), but

282 can also be influenced by processing at encoding. In young adults, false recognition is lower

283 when list items have unique as opposed to shared study context, in terms of either list blocking

284 (Goodwin, Meissner, \& Ericsson, 2001) or font (Arndt \& Reder, 2003). Such contextual effects

285 may be greater in older adults, as reflected in greater impact of blocking or task manipulations

286 (Taconnat, Clarys, Vanneste, \& Isingrini, 2006; Thomas \& Sommers, 2005; Tun et al., 1998; see

287 also Thomas, Bonura, \& Taylor, 2012). In the present study, although associated lists were

288 presented in the same fonts, it was possible that the judgement of specific "fit" of each unique

289 item to the font emphasized older adults' item-specific processing at the expense of relational

290 processing. If so, studying each word in a unique font should exaggerate this "reversed" age-

291 related difference.

\section{Experiment 2}

293

In this experiment, we contrasted false recognition of perceptually-related and

294 semantically-associated lures in a fully counterbalanced as well as matched design. Arndt and

295 Reder (2003) previously showed that adjusted critical lure false recognition was greater when list

296 items were studied in the same font (Correlated Font condition) than when they were each

297 studied in a different font (Unique Font; see Figure 1). We expected that if the age-related

298 reduction in critical lure false recognition in Experiment 1 was due to greater item-specific

299 encoding leading to reduced lure activation in the older adults, it would be more pronounced in 
300 the Unique Font relative to the Correlated Font conditions. If not, the logic used prior to

301 Experiment 1 predicted an increase in Correlated Font false recognition in the older group. We

302 also predicted that adjusted Font Only lure false recognition would again be greater in the older

303 adults. In both cases, we also expected age-related increases in false recollection.

\section{Method}

305 Participants

306 Participants were 24 young (18-33 years, $M=22 ; 17$ female) and 24 older (60-75 years,

$307 \mathrm{M}=67 ; 18$ female) adults. All were native English speakers with normal or corrected-to-normal

308 vision. As in Experiment 1, and as expected, the older group had higher crystallized IQ than the

309 young (WTAR standard score: $\mathrm{M}=110$ and $124, \mathrm{SD}=7$ and $3 ; \mathrm{t}(46)=8.52, \mathrm{p}<.001, \mathrm{~d}=2.5 ; 1$

310 missing value), and greater verbal fluency on the FAS Controlled Word Association Test (Lezak;

311 Howieson; Bigler; Tranel, 2012); $t(36.8)=6.52, \mathrm{p}<.001, \mathrm{~d}=1.9, \mathrm{M}=47$ and 62 words, $\mathrm{SD}=6$

312 and 10). Despite this, and as in Experiment 1, they showed a typical age-related decrement in

313 processing speed (Digit Symbol Coding completion time young $\mathrm{M}=202 \mathrm{sec}$, older $=239 \mathrm{sec}$,

$314 \mathrm{SD}=21$ and $35 ; t(38.0)=4.55, \mathrm{p}<.001, \mathrm{~d}=1.3)$ and were slower on part B relative to part A of

315 the Trail Making test (for difference $t(33.7)=2.49, \mathrm{p}=.018, \mathrm{~d}=.72 ; \mathrm{M}=22$ and $31 \mathrm{sec}, \mathrm{SD}=8$

316 and $16 \mathrm{sec})$. The groups did not differ significantly in Digit Span $(43.8)=1.98, \mathrm{p}=.054$,

317 although Bayesian evidence did not provide support for the null hypothesis either, $\mathrm{BF}_{01}=.72$ ).

318 Written consent was obtained from all participants. The study was approved by the Psychology

319 Research Ethics Committee, University of Edinburgh (ref. 80-1516/2).

320 Materials

321 Words were selected from 168 9-item DRM associate lists from the USF Free

322 Association Norms database (Nelson, McEvoy, \& Schreiber, 2004), and 157 distinctive fonts, 
323 also from the same sources as Experiment 1. The lists were selected to maximize BAS to their

324 critical lures $(M=0.32)$. In two conditions (see Figure 1 for design), sets of semantically

325 associated items were studied, and only Critical lures included at test (Correlated Font and

326 Unique Font). In the third, Font Only condition, sets of semantically unrelated items were again

327 studied in the same font, with unrelated lures in the same font at test, as in Experiment 1. In this

328 fully counterbalanced and matched design, we again used two types of Novel items to calculate

329 the baseline false alarm rates. For the Correlated Font and Unique Font conditions, in which

330 Critical lures were shown, the corresponding Novel items were critical lures from unstudied

331 DRM lists (Critical-matched). For the Font Only condition, in which lure words were (non-

332 critical) items from unstudied DRM lists, the Novel items were drawn from the same sets of

333 unrelated items as the studied items and lures (Related-matched).

334 Each study phase comprised 324 items, including 9 words from 12 associated DRM word

335 lists for the Correlated Font and Unique Font conditions, and 1 word from each of a further 84

336 associated word lists, grouped into sets of 9 for the Font Only condition (inspected to check they

337 were not obviously related). In the Correlated Font and Font Only conditions, all words in a 9-

338 item list (related or not) were presented in the same font (12 per condition). In the Unique Font

339 condition, each word was presented in a unique font ( 84 further fonts) (total $=324$ items). Each

340 test list comprised 98 items, including 36 studied words (one from each studied set in each of the

3413 conditions), 24 Critical lures (12 each for the Correlated Font and Unique Font conditions), and

34212 unrelated Font Only lures. Studied items were presented in the studied font in all conditions.

343 The Correlated Font Critical lures and the Font Only lures were also presented in the same font

344 as their corresponding studied items. The Unique Font Critical lures were presented in the same

345 font as one of their corresponding studied items. The 12 Critical-matched and 12 Related- 
346 matched Novel items were each presented in an unstudied font. Allocation of fonts and lists to

347 conditions was fully counterbalanced.

348 Procedure

349 The procedure was identical to that of Experiment 1.

350 Data analysis

351 The data analysis followed the same procedures as Experiment 1.

\section{Results}

Table 2 lists the raw and adjusted response proportions (collapsed over R and F), and

354 Figure $2(\mathrm{C}-\mathrm{D})$ illustrates the true and false recollection results.

$<$ Table 2 about here $>$

356 True recognition

Across both age groups participants were more likely to correctly recognize items from semantically associated lists which had been studied in the same font (Correlated Font) than those studied in unique fonts (Unique Font), or unrelated items which had been studied in the same font (Font Only) (Table 1 and Figure 2). For hits, ANOVA with factors of Condition and Group revealed only a main effect of condition, $F(2.0,90.8)=15.2$, MSE $=.27, \mathrm{p}<.001, \eta_{p}^{2}=$ .25 ; for Group main effect, $F(1,46)=.03, \mathrm{MSE}=.001, \mathrm{p}=.87, \mathrm{BF}$ against inclusion in the model $=4.1$; for interaction, $F(2.0,90.8)=.25, \mathrm{MSE}=.004, \mathrm{p}=.78, \mathrm{BF}=9.1$ against inclusion

364 in the model. Post hoc tests confirmed differences between Correlated Font and both the Unique

365 Font and the Font Only conditions, $t(47)=4.47, \mathrm{p}<.001, \mathrm{~d}=.65$ and $t(47)=-5.12, \mathrm{p}<.001, \mathrm{~d}=$ 366.74 , but not between the Unique Font and Font Only conditions, $t(47)=1.02, \mathrm{p}=.314$; alpha $=$ $367.017, \mathrm{BF}_{01}$ for null hypothesis $=3.9($ range $=2.9$ for prior width $=.5$ to 4.9 for width .9$)$.

368 Analysis of adjusted true recognition (using false alarms to the "unrelated" Novel items drawn 
369 from unstudied DRM lists) showed the same pattern with a significant main effect of Condition

370 only, $F(2.0,90.1)=15.3, \mathrm{MSE}=.271, \mathrm{p}<.001, \eta_{\mathrm{p}}^{2}=.25$, and non-significant Group main

371 effect, $F(1,46)=2.54, \mathrm{MSE}=.17, \mathrm{p}=.118$, although only anecdotal Bayesian evidence for the

372 null, $\mathrm{BF}_{01}=1.3$. The pattern was the same for true recollection (for main effect of Condition,

$373 F(1.9,85.4)=15.7, \operatorname{MSE}=.41,, \mathrm{p}<.001, \eta_{\mathrm{p}}^{2}=.25 ;$ for Group, $F(1,46)=3.3, \mathrm{MSE}=.29, \mathrm{p}=$

$374.078, \mathrm{BF}$ for inclusion in the model $=1.1$; for interaction, $F(11.9,85.6)=.67, \mathrm{MSE}=.02, \mathrm{BF}$

375 against inclusion in the model $=2.5$ ). Therefore, as in Experiment 1, older adults' true

376 recognition was similar to that of the young. Participants in both groups were more likely to

377 recognize studied items which were semantically associated with others on the list than those

378 only printed in the same font. We also did not find evidence for (or clearly against) an age-

379 related boost to true memory from the semantic relations.

380 False recognition

381 Baseline false recognition of novel items was higher for Critical-Matched than Related-

382 matched items, with no difference between age groups (Table 2 and Fig 2). ANOVA with factors

383 of Condition and Group showed only a main effect of condition, $F(1,46)=4.7$, MSE $=.031, \mathrm{p}=$

$384.036, \eta_{\mathrm{p}}^{2}=.09$. Although the main effect of Group was not significant, $\mathrm{F}(1,46)=.862$, MSE $=$

$385.028, \mathrm{p}=.358 ; F(1,46)=3.24, \mathrm{MSE}=.021, \mathrm{p}=.079$, Bayesian ANOVA did not show evidence

386 against inclusion of this factor either, $\mathrm{BF}_{01}=1.1$. Proportions of novel items which were falsely

387 recollected did not differ significantly according to Group or Condition. The possibility of subtle

388 group differences in Novel FA might complicate interpretation of Novel-adjusted false

389 recognition measures as in Experiment 1. However, unlike in Experiment 1, findings from the

390 different measures converged, as outlined below. 
392 the two other conditions, but the groups also differed according to condition (Table 2 and Fig 2).

393 ANOVA with factors of Condition and Group showed a significant interaction, $F(1.9,89.2)=$

$3945.6, \mathrm{MSE}=.15, \mathrm{p}=.006, \eta_{p}^{2}=.11$, as well as main effects of Condition, $F(1.9,89.2)=78.3$,

$395 \mathrm{MSE}=2.1, \mathrm{p}<.001, \eta_{p}^{2}=.63$, and Group, $\left.F(1,46)=9.3, \mathrm{MSE}=.56, \mathrm{p}=.004, \eta^{2}=.17\right)$. Post

396 hoc tests showed significant age-related differences in the Font Only Condition, $\mathrm{t}(40.0)=-3.99$,

$397 \mathrm{p}<.001 ; \mathrm{d}=1.15$ and the Unique Font condition, $\mathrm{t}(37.3)=-2.6, \mathrm{p}=.013, \mathrm{~d}=.75 ;$ alpha $=.017$,

398 but not in the Correlated Font condition, $\mathrm{t}(39.7)=-.15, \mathrm{p}=.88$, in which there was moderate

399 evidence in favour of the null, $\mathrm{BF}_{01}=3.4$ (range $=2.67$ for prior width $=.5,4.3$ for prior width $=$

400 .9. This replicated the results of Experiment 1 for the two shared conditions, showing evidence

401 against an age-related increase in associatively-driven false recognition because the groups did

402 not differ for the Correlated Font lures. At the same time, there was again an increase in false

403 recognition of Font Only lures in older adults. So far, findings were consistent with a greater

404 tendency to perceptually-driven false recognition, but not semantically-driven false recognition, 405 in older adults.

406 The false recollection results were similar to those for raw FA, except that the groups did 407 not differ in the Unique Font condition. ANOVA showed an interaction of Condition x Group, $408 F(1.6,74.6)=8.78, \mathrm{MSE}=.16, \mathrm{p}<.001, \eta_{p}^{2}=.16$, and $p o s t$ hoc tests showed group differences 409 for Font Only, $t(36.9)=-3.91, \mathrm{p}<.001, \mathrm{~d}=1.13$, but not for Correlated Font, $\mathrm{t}(44.5)=.56, \mathrm{p}=$ $410.58, \mathrm{BF}_{01}=3.1($ range $=2.4$ for prior width $=.5$ to 3.7 for width .9$)$, or Unique Font, $\mathrm{t}(45.2)=-$ $411.11, \mathrm{p}=.92 ;$ alpha $=.017 ; \mathrm{BF}_{01}=3.5($ range $=2.7$ for prior width .5 to 4.2 for width .9$)$. Again, 412 these findings converged with those of Experiment 1 for the two shared conditions. 
414 and Font Only conditions, after correcting for baseline false alarms to Novel items (Fig 1),

415 converged with those for overall false recognition and false recollection. ANOVA showed main 416 effects of Condition, $F(2.0,90.0)=53.1, \eta_{\mathrm{p}}^{2}=.54, \mathrm{MSE}=1.90, \mathrm{p}<.001$, and Group, $F(1,46)=$

$4177.07, \mathrm{MSE}=.36, \mathrm{p}=.011, \eta_{\mathrm{p}}^{2}=.13$. There was no clear evidence for an interaction, $F(2.0,90.0)$ $418=2.51, \mathrm{MSE}=.09, \mathrm{p}=.088, \mathrm{BF}$ for inclusion in the model $=2.5$. Therefore, the older adults 419 showed generally greater false recognition after correction for novel item false alarms, and the 420 conditions did not differ. Age effects were also robust in the Font Only condition taken alone, consistent with Experiment 1's findings and our prediction of an age-related increase in

422 perceptually-driven false recognition based on findings from the categorized pictures paradigm 423 (Pidgeon \& Morcom, 2014). Pairwise post hoc tests (alpha =.017) showed no group difference 424 in the Correlated Font condition, $t(45.1)=.07, \mathrm{p}=.95 ; \mathrm{BF}_{01}$ in favour of null hypothesis $=3.5$ 425 (range $=2.7$ with prior width .5 to 4.3 with width .9 ), no clear evidence for or against a group 426 difference in the Unique Font conditions, $t(41.3)=-2.28, \mathrm{p}=.028 ; \mathrm{BF}_{01}=.45$, but significantly 427 higher false recognition in the older than the young adults in the Font Only condition, $t(45.6)=$ 428 $4.41, \mathrm{p}<.001, \mathrm{~d}=1.3$.

429 False recognition across experiments 1 and 2 To check the apparent convergence of the principal findings across the two experiments, 431 we calculated across-experiment Bayes factors for comparisons of age-related differences in 432 false recollection in the two shared conditions. We focus on the proportions of items falsely 433 judged recollected, which were directly comparable across experiments and not biased by any 434 differences in novel item processing. This measure is also indicative of effects on vivid false 435 recollection rather than only on familiarity or guessing (the results were qualitatively similar for 
436 analyses of raw false recognition measures). As noted above, Bayes factors provide

437 complementary information to $\mathrm{p}$ values, indicating the strength of evidence for the null or the

438 alternative hypothesis. A Bayes factor is also not affected by the number of statistical tests

439 performed, unlike $\mathrm{p}$ values (Wagenmakers, 2007). We found extreme evidence for greater false

440 recollection of Font Only lures in the older relative to the young adults across experiments, $\mathrm{BF}_{10}$

441 against the null hypothesis $=299.6$ (range 278 for prior width .5 to 297 for width .9 ), $95 \%$

442 credible interval for false recollection proportions in young $=.03$ to .08 with $\mathrm{M}=.053$; in older $=$

443.13 to .26 with $\mathrm{M}=.198$. However, for Correlated critical lures there was evidence for the null

444 hypothesis of no age-related difference in false recollection, $\mathrm{BF}_{01}$ for null hypothesis $=3.9$, range

$445=2.9$ for prior width .5 to 4.8 for width $.9,95 \%$ credible intervals for young $=.31$ to .41 , for

446 older $=.26$ to .41 . When the two measures were entered into a Bayesian ANOVA with factors of

447 Condition (Correlated/ Font Only), Group (Young/ Older) and Experiment (1/ 2), the Bayes

448 factor in favor of inclusion of the interaction of Condition with Group was 2055.9. We did not

449 find evidence for a difference between experiments in this effect, nor against one, with $\mathrm{BF}=.84$

450 against inclusion of the 3-way interaction. These results were qualitatively unchanged after

451 exclusion of 3 older individuals whose overall false alarm rates were very high ( $>.5$ for one or

452 more Novel or Lure conditions; $\mathrm{BF}_{10}=187.9$ for an age-related increase in Font Only RFA, $\mathrm{BF}_{10}$

$453=445.8$ for Group $\mathrm{x}$ Condition, and $\mathrm{BF}_{10}=1.1$ for Group $\mathrm{x}$ Condition $\mathrm{x}$ Experiment). Together,

454 the data therefore strongly supported our original hypothesis that older adults would show

455 greater perceptually-driven false recollection (in the Font Only conditions). They also suggested

456 that across experiments, false recollection due to associative semantic relations in this task was

457 age-invariant.

458 Discussion 
460 age-related increase in lure false recognition. In this fully counterbalanced and matched design,

461 in which item differences between novel and lure conditions could not contaminate age-related

462 comparisons, we found converging results between the adjusted false recognition and the raw FA

463 and false recollection measures. There was no hint of Experiment 1's 'reversed' age-related

464 difference for the critical lures on the adjusted measure. Although age effects did not differ

465 between conditions, it is notable that while the age-related increase in Font Only lure false

466 recognition was clear cut, there was no evidence of an age effect on semantically-driven false

467 recognition.

468 The results for the Unique Font condition were less clear than for the other two

469 conditions, and are discussed further below. The main reason to include this condition was our

470 prediction that if an age-related reduction in associative false recognition were again found in the

471 Correlated Font condition in the second experiment, this reduction might reflect greater item-

472 specific processing in older adults, expected to be enhanced further and therefore more

473 pronounced for the Unique Font lures. Instead, there was no significant age effect in the Unique

474 Font condition (nor clear evidence against one): an increase in false recognition in the older

475 group was not robustly present after adjustment for baseline false alarms, nor present for false

476 recollection responses.

\section{General Discussion}

In these experiments, older adults were more prone to perceptual false recognition than

479 the young, and showed an increase in perceptual false recollection, but we did not find any

480 increase in associative false recognition. The data are contrary to the general hypothesis that

481 older adults falsely recognize more than the young because their episodic memory relies more on 
482 prior knowledge (Koutstaal et al., 2003; Pidgeon \& Morcom, 2014; Reder et al., 2007). On this

483 hypothesis, a greater age-related increase in false recognition was expected in the Correlated

484 Font compared to the Font Only conditions. Instead, our results suggest that prior knowledge - in

485 this case associations - is not always the main driver of older adults' memory errors, and that

486 perceptual information plays a role. We also found that these errors were accompanied by

487 recollective experience, as predicted and in line with other evidence that older adults' memory

488 errors are often accompanied by subjective recollection (see Devitt \& Schacter, 2016; McCabe et

489 al., 2009).

490 In the present study, older adults were about twice as likely as the young to falsely

491 recognize unrelated lure words which were written in the same font as multiple studied words,

492 and almost four times as likely to falsely recollect these lures. The data converge with our

493 previous finding that older adults were more likely to falsely recognize lure pictures from pre-

494 experimentally unfamiliar 'abstract' object categories (Pidgeon \& Morcom, 2014). Both in that

495 study and the present study, the age-related increase in false recognition was only observed for

496 lures which resembled multiple studied items (see also Koutstaal et al., 2003; Koutstaal,

497 Schacter, Brenner, \& Jackson, 1999; Koutstaal \& Schacter, 1997). In our earlier study, one

498 possibility is that this false recognition, and its increase in aging, was driven by the object-like

499 nature of the 'abstract' images, some of which we found to be consistently nameable (Pidgeon \&

500 Morcom, 2014). Perceptual influences on false recognition are not well understood, but it is

501 recognized that some form of prototype or gist extraction can proceed without prior knowledge,

502 as shown by false recognition of abstract dot patterns which are similar to sets of studied patterns

503 (Posner \& Keele, 1970; Gallo, 2006, pp 35-36). However, apparent perceptual gist effects can

504 also reflect de novo category learning that proceeds as multiple exemplars are encountered 
505 (Stahl, Henze, \& Aust, 2017, May 15). Potentially, some kind of conceptual representation might

506 form for individual novel fonts in the present task. However, false recognition of pictures of

507 objects resembling only single studied exemplars has also been demonstrated, supporting the

508 suggestion that perceptual similarity itself can drive false recognition (Stahl et al., 2017, May 509 15).

510 Support for increased perceptually-driven false recognition in aging also comes from 511 several studies where multiple studied words are related to phonologically similar lures (Budson

512 et al., 2003; Sommers \& Huff, 2003; Watson et al., 2001; see also Rankin \& Kausler, 1979),

513 although not in a recent study in which lures were only related to single studied words (Ly,

514 Murray \& Yassa, 2013). As for pictures, an age-related increase in false recognition of

515 perceptually similar verbal lures may be present only when multiple related items have been

516 studied. However, for phonological lures this is unlikely to be driven by formation of a novel

517 conceptual representation, or by idiosyncratic categorical processing. The present data extend

518 these findings by demonstrating greater false recognition of visually similar lures in older adults

519 under conditions in which multiple items have been studied. They support the notion that older

520 adults are more sensitive to recognition based on perceptual gist representations of sets of related

521 but - unlike in the case of the phonologically similar lures - pre-experimentally unfamiliar

522 features.

523 In contrast to the findings just discussed for the Font Only condition, we found age-

524 invariant associative false recognition, both in Experiment 2 alone and across the two

525 experiments. This invariance was supported by Bayes Factors for null effects of age in the

526 Correlated Font condition. Critically, false recognition by older adults was not greater in this

527 condition than in the Font Only condition, as originally predicted. Although older adults often 
528 show increased false recognition in the DRM paradigm, a number of previous studies have also

529 found no such effect. Across studies published prior to 2006 in which recognition was not

530 measured after an initial recall test, Gallo (2006) found a non-significant (3\%) mean increase in

531 false recognition, although there was a significant (7\%) mean increase in false recall. A key

532 factor determining whether there are age effects is thought to be impairments in retrieval

533 monitoring, demands for which are greater for recall than recognition and which may also be

534 encouraged by list blocking (Gallo, 2006). A second modifying factor is thought to be the degree

535 to which the experimental task encourages reliance on gist as opposed to specific memory

536 (Koutstaal, 2006; Tun, Wingfield, Rosen, Blanchard, \& Tun, 1998). Tun et al. (1998) found that

537 older adults were more susceptible to false recognition only when study lists were blocked,

538 unlike in the present study. Although our finding of age-invariant associative false recognition

539 does not rule out age-related increases in blocked tasks, it is notable that we still found an age-

540 related increase in false recognition of perceptually related lures.

541 In Experiment 2, the results for the Unique Font condition (in which lures differed in

542 their font from all but one studied associate) were inconclusive. Overall and adjusted false

543 recognition measures showed non-significant (but not clearly null) age effects which were

544 intermediate between those for the Correlated Font (in which lures were similar in terms of their

545 font to all studied associates) and Font Only conditions. This was not the clear pattern of age-

546 invariant performance observed in the Correlated Font condition and therefore limits the

547 generality of our conclusion that associative false recognition was not increased in older people

548 in the present study. It will be important in future studies to compare age effects in a Correlated

549 Font condition with those in a DRM condition with a standard simple font. 
Another potential influence on both semantically- and perceptually-related false

551 recognition in older adults is differential responding to the font information between age groups.

552 One possibility is that older adults misunderstood the instructions and responded instead to

553 identify test items in familiar fonts as studied. This strategy would have given rise to very high

554 false alarm rates in older adults in the Correlated Font as well as the Font Only condition, but not

555 in the Unique Font condition in Experiment 2. Under these conditions, we should have observed

556 age-related increases in adjusted false recognition in the Correlated Font and Font only

557 conditions relative to the Unique Font condition, a pattern not supported by the data. Extreme

558 values of adjusted false recognition were also not markedly more common in the older group: in

559 Experiment 1, 3 young and 4 older showed values of $>=.5$, and in Experiment 2, 7 young and 8

560 older showed FA values of $>=.75$ (see Tables 1 and 2). Three older individuals across the two

561 studies did show high overall FA (to Novel as well as to studied-font conditions), but excluding

562 them from analysis did not change the overall pattern of findings (see False recognition across

563 experiments). Another possibility was group differences in the ability to avoid memory errors by

564 systematically selectively attending to one dimension of the stimuli. In the present paradigm,

565 because the perceptual similarity between words printed in the same fonts is task-irrelevant in the

566 test phase, it would be beneficial to ignore the fonts as far as possible, and emphasize processing

567 of the words' identity alone. Older adults may have been less able to do this, consistent with

568 evidence that they often fail to suppress irrelevant information (Hasher, Quig \& May, 1997;

569 Campbell, Hasher \& Thomas, 2010; Devitt \& Schacter, 2016). In the categorized pictures

570 paradigm, on the other hand, it is advantageous to attend to the perceptual information. However,

571 despite this difference, the present data together with those of Pidgeon and Morcom (2014) 
572 suggest that there is greater perceptually-related false recognition in older than young adults in

573 both paradigms (see below).

574 If older adults are always more susceptible to perceptual similarity between lures and

575 studied items, the age-related increase in false recognition in the Correlated Font condition

576 should have been as large or larger than in the Font Only condition, at least if associative and

577 perceptual effects are independent. Both age groups falsely recognized more Correlated Font

578 lures than Unique Font lures, replicating Arndt and Reder's (2003) results despite the intermixed

579 presentation. However, shared font did not boost false recognition of critical lures specifically in

580 older adults. One reason for this could be that older adults did not process font information as

581 strongly when items were associatively related. Although encoding processing was presumably

582 similar since conditions were intermixed, it is possible that in older adults, rapid high-confidence

583 recognition of the critical lures at retrieval led to reduced incidental processing of font

584 information. A second, perhaps more plausible, possibility derives from the finding that in young

585 adults, false recognition of critical lures is specifically enhanced when they are presented in the

586 same font as their studied associates, as opposed to when they are presented in the same font as

587 other studied items (Arndt, 2010). This must reflect encoding of the association between font and

588 study list, and there is strong evidence that associative memory is impaired in aging (Naveh-

589 Benjamin, 2000). Thus, older adults' false recognition may have lacked this boost. Note that both

590 these proposals contradict the above suggestion that young adults may have been better at

591 ignoring font information at test.

592 Our starting point was a comparison with earlier work using the categorized pictures

593 paradigm (Koutstaal et al., 2003; Pidgeon and Morcom, 2014). The present results suggest that

594 the determinants of older adults' reliance on gist and/or prior knowledge may differ from those 
595 in the DRM paradigm. To our knowledge, all published studies have found substantial increases

596 in older adults' false recognition of categorically-related lures, even though conditions are

597 intermixed at encoding and retrieval (e.g., Koutstaal, Schacter, Galluccio, \& Stofer, 1999;

598 Koutstaal, 2003; Koutstaal et al., 2003; Koutstaal \& Schacter, 1997; Lovden, 2003; Pidgeon \&

599 Morcom, 2014; Rankin \& Kausler, 1979). Furthermore, older adults show greater increases in

600 false recognition of lures from familiar 'concrete' categories than of unfamiliar 'abstract'

601 categories (Koutstaal et al., 2003; Pidgeon and Morcom, 2014). It is therefore possible that prior

602 semantic knowledge does specifically impact memory errors in older adults but does so

603 predominantly via similarity, rather than via association. Older adults are also more likely to

604 falsely recognize phonologically-similar lures than the young (although in those studies older

605 groups also showed increased associative false recognition, unlike here; Budson et al., 2003;

606 Watson et al., 2001). Another factor differing between DRM and categorized pictures paradigms

607 may be the relative influences of the multiple types of semantic and associative relations between

608 studied items and lures which have been shown to contribute to false recognition (e.g. Cann,

609 McRae, \& Katz, 2011; Coane, McBride, Termonen, \& Cutting, 2015; McEvoy, Nelson, \&

610 Komatsu, 1999; Montefinese, Zannino, \& Ambrosini, 2015). Systematic evaluation of these

611 variables in needed to understand the specific mediators of the sometimes very pronounced

612 effects of age on false memory.

\section{Conclusions}

614 The present study examined false recognition of associatively related and perceptually

615 similar lures in older and younger adults. While older adults did not show an increase in false

616 recognition of critical lures, they were more likely to falsely recognize lures which were printed

617 in the same font as studied items. The results suggest that increases in false memory in aging are 
618 not always driven by greater reliance on prior knowledge, but that older adults show increased

619 false recollection which can reflect increased susceptibility to perceptual resemblance between

620 novel and previously encountered occurrences.

\section{Acknowledgements}

The authors are grateful to Rebecca Williams for assistance with data collection. 


\section{References}

622 Arndt J. 2010. The role of memory activation in creating false memories of encoding context.

623 Journal of Experimental Psychology: Learning, Memory, and Cognition 36:66-79. DOI:

$624 \quad 10.1037 / \mathrm{a} 0017394$.

625 Brainerd, J., \& Reyna, V. F. (2002). Fuzzy-Trace Theory and False Memory. Current Directions 626 in Psychological Science, 11(5), 164-169.

627 Budson AE., Sullivan AL., Daffner KR., Schacter DL. 2003. Semantic versus

628 phonological false recognition in aging and Alzheimer's disease. Brain and Cognition 51:251-

629 261. DOI: 10.1016/S0278-2626(03)00030-7.

630 Campbell KL., Hasher L., Thomas RC. 2010. Hyper-binding: a unique age effect. Psychological 631 science 21:399-405. DOI: 10.1177/0956797609359910.

632 Castel AD. 2005. Memory for grocery prices in younger and older adults: the role of schematic 633 support. Psychology and aging 20:718-721. DOI: 10.1037/0882-7974.20.4.718.

634 Devitt AL., Schacter DL. 2016. False memories with age_neural and cognitive underpinnings. 635 Neuropsyhology 91:346-359. DOI: 10.1016/j.neuropsychologia.2016.08.030.

636 Gallo, DA. 2006. Associative Illusions of Memory. New York: Psychology Press.

637 Hasher L., Quig MB., May CP. 1997. Inhibitory control over no-longer-relevant information: 638 adult age differences. Memory \& cognition 25:286-95. DOI: 10.3758/BF03211284.

639 Kensinger EA., Schacter DL. 1999. When true memories suppress false memories: effects of $640 \quad$ aging. 16:399-415.

641 Koutstaal W. 2003. Older adults encode — but do not always use — perceptual details: 
644 Koutstaal W. 2006. Flexible remembering. Psychonomic bulletin \& review 13:84-91. DOI: 10.3758/BF03193817.

646 Koutstaal W., Reddy C., Jackson EM., Prince S., Cendan DL., Schacter DL. 2003. False 647 recognition of abstract versus common objects in older and younger adults: testing the 648 semantic categorization account. Journal of experimental psychology. Learning, memory, 649 and cognition 29:499-510. DOI: 10.1037/0278-7393.29.4.499.

650 Koutstaal W., Schacter DL. 1997. Gist-Based False Recognition of Pictures in Older and $651 \quad$ Younger Adults. Journal of Memory and Language 37:555-583. DOI:

652 10.1006/jmla.1997.2529.

653 654 655 656 657 658

Koutstaal W., Schacter DL., Brenner C., Jackson EM. 1999a. Perceptually based false recognition of novel objects in amnesia: effects of category size and similarity to category. Cognitive neuropsychology 16:317-341.

Koutstaal W., Schacter DL., Galluccio L., Stofer K a. 1999b. Reducing gist-based false recognition in older adults: encoding and retrieval manipulations. Psychology and aging 14:220-237. DOI: 10.1037/0882-7974.14.2.220.

Lee MD., Wagenmakers E-J. 2013. Bayesian cognitive modeling: A practical course. Cambridge University Press.

Lezak, M D; Howieson, D B; Bigler, E D; Tranel D. 2012. Neuropsychological Assessment. New York: Oxford University Press.

Lovden M. 2003. The episodic memory and inhibition accounts of age-related increases in false memories: A consistency check. Journal of Memory and Language 49:268-283. DOI: 
666 Ly M., Murray E., Yassa MA. 2013. Perceptual versus conceptual interference and pattern 667 separation of verbal stimuli in young and older adults. Hippocampus 23:425-430. DOI: $668 \quad$ 10.1002/hipo.22110.

669 Mandler G. 1980. Recognizing: The judgment of previous occurrence. Psychological Review $670 \quad 87: 252-271$. DOI: $10.1037 / 0033-295 X .87 .3 .252$.

671 McCabe DP., Roediger HL., Mcdaniel MA., Balota DA. 2009. Neuropsychologia Aging reduces 672 veridical remembering but increases false remembering : Neuropsychological test correlates 673 of remember - know judgments. 47:2164-2173. DOI:

$674 \quad$ 10.1016/j.neuropsychologia.2008.11.025.

675 Migo EM., Mayes AR., Montaldi D. 2012. Measuring recollection and familiarity: Improving the 676 remember/know procedure. Consciousness and Cognition 21:1435-1455. DOI:

677 10.1016/j.concog.2012.04.014.

678 Naveh-Benjamin M. 2000. Adult Age Differences in Memory Performance: Tests of an 679 Associative Deficit Hypothesis. 26:1170-1187. DOI: 10.1037//0278-7393.26.5.1170.

680 Naveh-Benjamin M., Hussain Z., Guez J., Bar-On M. 2003. Adult age differences in episodic 681 memory: further support for an associative-deficit hypothesis. Journal of experimental 682 psychology. Learning, memory, and cognition 29:826-837. DOI: 10.1037/0278$683 \quad 7393.29 .5 .826$.

684 Norman KA., Schacter DL. 1997. False recognition in younger and older adults: exploring the 685 characteristics of illusory memories. Memory \& cognition 25:838-48. DOI:

686 10.3758/bf03211328. 
687 Pidgeon LM., Morcom AM. 2014. Age-related increases in false recognition: The role of 688 perceptual and conceptual similarity. Frontiers in Aging Neuroscience 6. DOI: $689 \quad 10.3389 /$ fnagi.2014.00283.

690 Rankin JL., Kausler DH. 1979. Adult Age Differences in False Recognitions. 34:58-65.

691 Reitan RM. 1958. Validity of the Trail Making Test as an indicator of organic brain damage. $692 \quad$ Perceptual and Motor Skills 8:271-276.

693 Salthouse TA., Atkinson TM., Berish DE. 2003. Executive Functioning as a Potential Mediator 694 of Age-Related Cognitive Decline in Normal Adults. Journal of Experimental Psychology: 695 General 132:566-594. DOI: 10.1037/0096-3445.132.4.566.

696 Schacter DL., Israel L., Racine C. 1999. Suppressing False Recognition in Younger and Older 697 Adults: The Distinctiveness Heuristic. Journal of Memory and Language 40:1-24. DOI: 698 10.1006/jmla.1998.2611.

699 Schacter DL., Koutstaal W., Norman KA. 1997. False memories and aging. Trends in Cognitive $700 \quad$ Sciences 6613:229-236.

701 Sommers MS., Huff LM. 2003. The effects of age and dementia of the Alzheimer's type on 702 phonological false memories. Psychology and aging 18:791-806. DOI: 10.1037/0882$703 \quad 7974.18 .4 .791$.

704 Stahl C., Henze L., Aust F. False memory for perceptually similar but conceptually distinct line 705 drawings.

706 Tun P a., Wingfield A., Rosen MJ., Blanchard L., Tun A. 1998. Response latencies for false 707 memories: gist-based processes in normal aging. Psychology and aging 13:230-241. DOI: 708 10.1037/0882-7974.13.2.230. 
709 Umanath S., Marsh EJ. 2014. Understanding How Prior Knowledge Influences Memory in Older

$710 \quad$ Adults. Perspectives on Psychological Science 9:408-426. DOI:

$711 \quad 10.1177 / 1745691614535933$.

712 Watson JM., Balota DA., Sergent-Marshall SD. 2001. Semantic, phonological, and hybrid

713 veridical and false memories in healthy older adults and in individuals with dementia of the

714 Alzheimer type. Neuropsychology 15:254-267. DOI: 10.1037//0894-4105.15.2.254.

715 Wilson IA., Gallagher M., Eichenbaum H., Tanila H. 2006. Neurocognitive aging: prior

716 memories hinder new hippocampal encoding. Trends in Neurosciences 29:662-670. DOI:

$717 \quad$ 10.1016/j.tins.2006.10.002. 


\section{Figure 1}

Design figure (new)

Design of Experiments 1 and 2. Panel A: partial example sets of studied words for each condition (see Materials). Panel B: example studied and lure test words corresponding to these studied sets for each condition, and novel items. * indicates a condition used only in Experiment 1. ** indicates a condition used only in Experiment 2. 
A

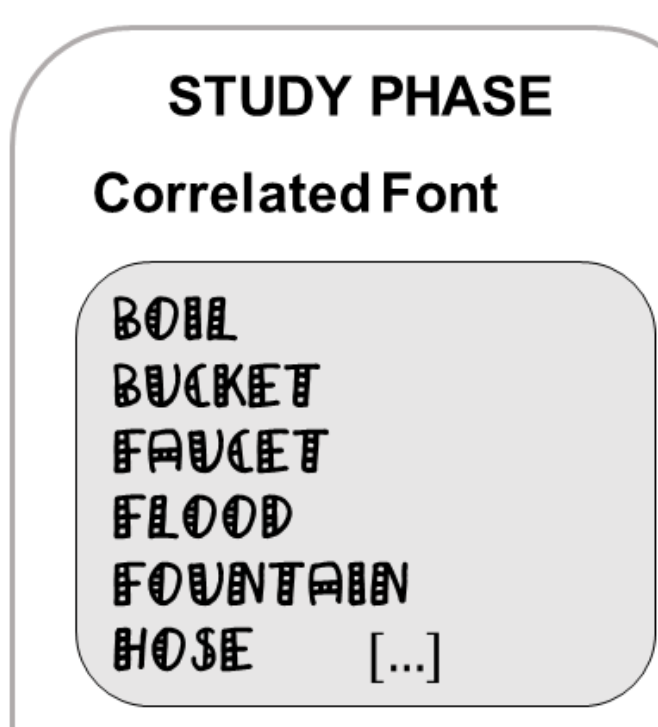

\section{Unique Font ${ }^{* *}$}

PASTERE

C ATPLE

MICLIS

SEN

BQBO

F周阳

$[\ldots]$

\section{Font Only}

EWGTWE

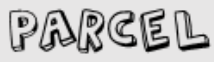

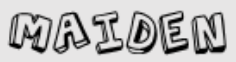

F०Wس

EEASTLY

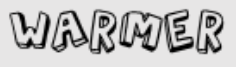

B

\section{TEST PHASE}

Correlated Font

BO囬 Studied

M国聑国 Critical lure

且目Q目睓 Related lure*

\section{Unique Font ${ }^{\star *}$}

PASTUERE Studied

Critical lure

Font Only

PARERL Studied

FASP Critical lure

Novel Items

BOTCH Critical-matched

PLAIE

Related-matched 
Figure 2

True and False Recollection.

Panels A (False Recollection) and B (True Recollection) show data from Experiment 1, and Panels C (False Recollection) and D (True Recollection) show data from Experiment 2. True Recollection is the proportion of studied items which were (correctly) recollected. False Recollection is the proportion of unstudied lures which were (incorrectly) recollected. Bars show the mean proportions of recollected items, and error bars represent SE.

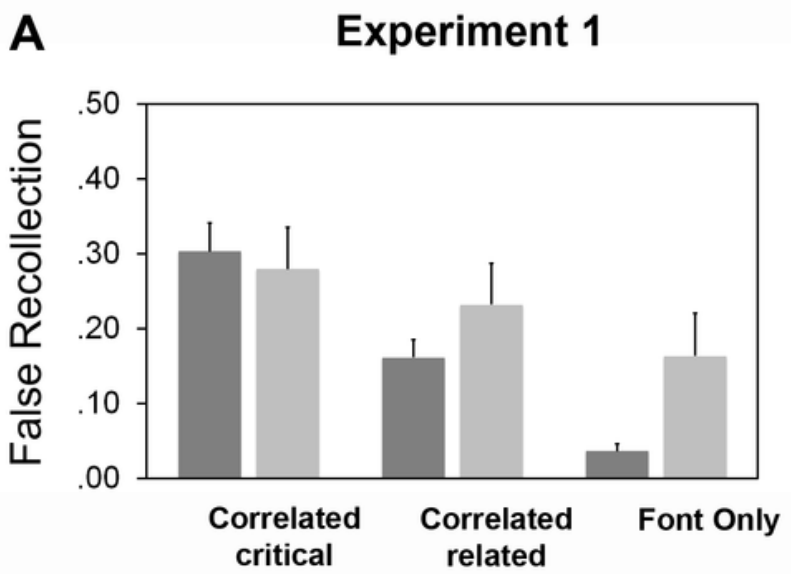

C Experiment 2

B
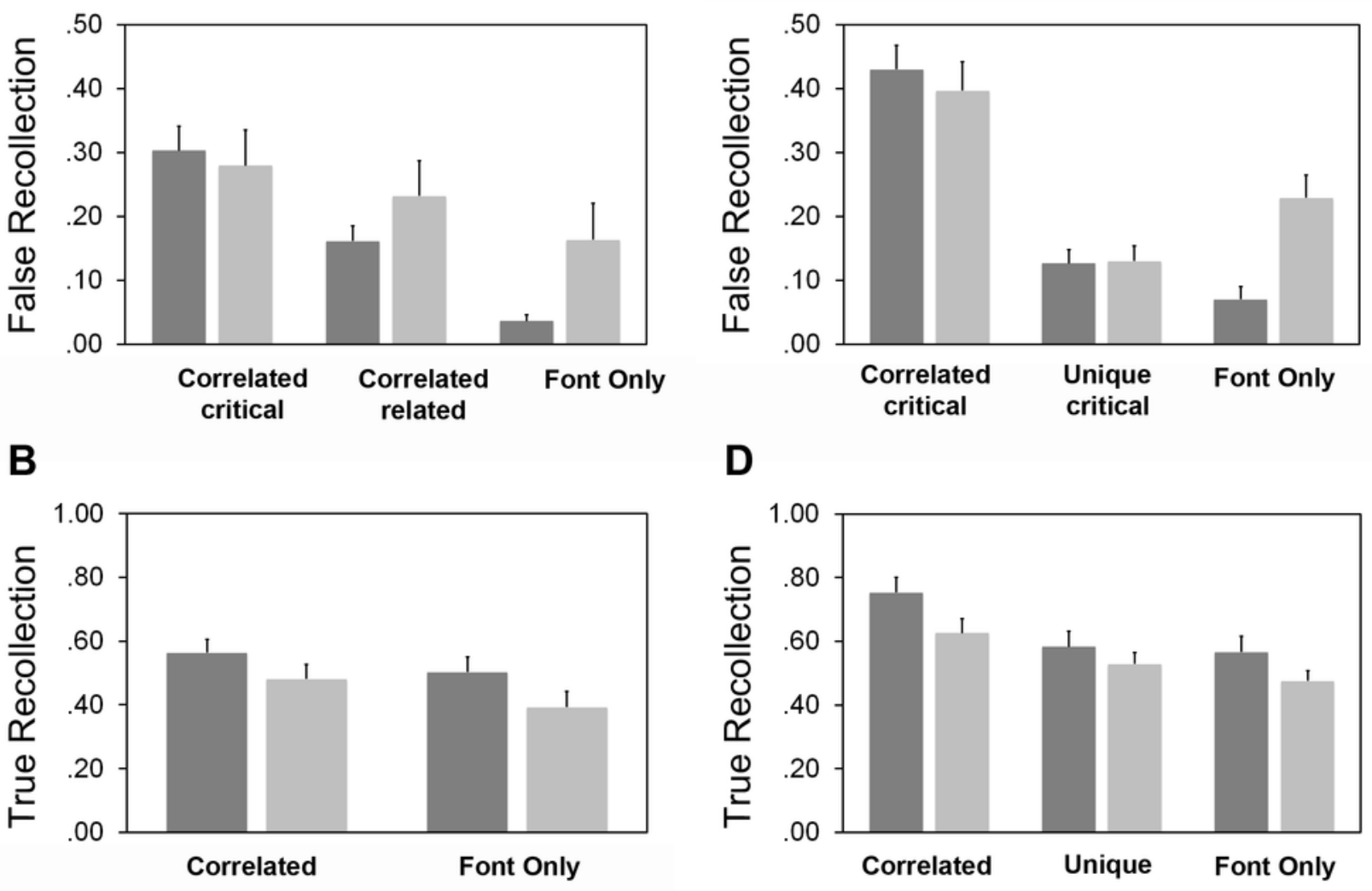

D

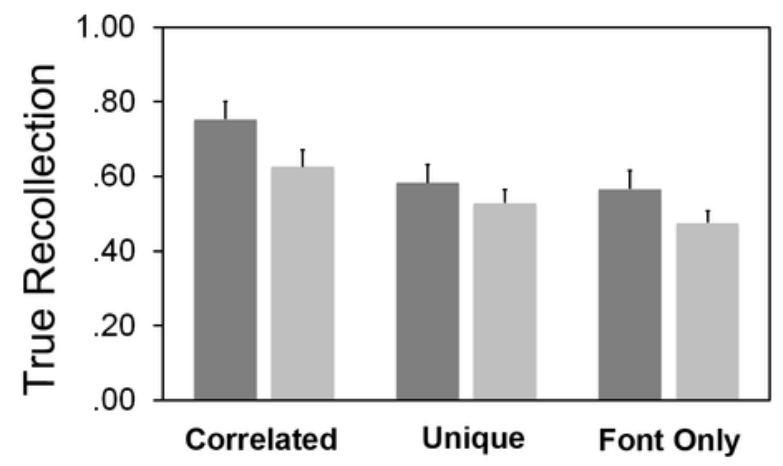

YOUNG

OLDER 


\section{Table $\mathbf{1}$ (on next page)}

True and False Recognition as a function of age, condition, and item type in Experiment 1.

(Means, with SD in parentheses). Overall Recognition proportions are the proportions of items in each condition which were judged old (both "Remember" and "Familiar" responses). Adjusted recognition scores are the raw Overall scores after subtraction of the raw scores for the corresponding Novel item condition (see Method for details of conditions and measures). 


\begin{tabular}{llccc}
\hline & Overall Recognition & \multicolumn{2}{c}{ Adjusted Recognition } \\
\hline Correlated Font & Young & Older & Young & Older \\
Studied item true recognition: & $.79(.14)$ & $.74(.17)$ & $.75(.16)$ & $.66(.30)$ \\
List lure false recognition: & $.34(.17)$ & $.45(.29)$ & $.29(.18)$ & $.30(.23)$ \\
Critical lure false recognition: & $.57(.19)$ & $.52(.28)$ & $.53(.18)$ & $.36(.24)$ \\
Font Only & & & & \\
Studied item true recognition: & $.70(.17)$ & $.65(.24)$ & $.59(.14)$ & $.50(.25)$ \\
Lure false recognition: & $.14(.11)$ & $.30(.29)$ & $.09(.10)$ & $.15(.18)$ \\
Novel & & & & \\
List-Matched false recognition: & $.04(.06)$ & $.15(.28)$ & \\
Critical-Matched false recognition: & $.04(.08)$ & $.16(.26)$ & \\
\hline
\end{tabular}




\section{Table 2 (on next page)}

True and False Recognition as a function of age, condition, and item type in Experiment 2.

(Means, with SD in parentheses). Overall Recognition proportions are the proportions of items in each condition which were judged old (both "Remember" or "Familiar" responses). Adjusted recognition scores are the raw Overall scores after subtraction of the raw scores for the corresponding Novel item condition (see Method for details of conditions and measures). 


\begin{tabular}{|c|c|c|c|c|}
\hline & \multicolumn{2}{|c|}{ Overall Recognition } & \multicolumn{2}{|c|}{ Adjusted Recognition } \\
\hline & Young & Older & Young & Older \\
\hline \multicolumn{5}{|l|}{ Correlated Font } \\
\hline Studied hits: & $.91(.14)$ & $.88(.11)$ & $.87(.14)$ & $.78(.18)$ \\
\hline Critical lure false alarms: & $.71(.15)$ & $.71(.23)$ & $.61(.20)$ & $.61(.23)$ \\
\hline \multicolumn{5}{|l|}{ Unique Font } \\
\hline Studied hits: & $.78(.18)$ & $.78(.15)$ & $.75(.21)$ & $.68(.20)$ \\
\hline Critical lure false alarms: & $.27(.13)$ & $.41(.22)$ & $.18(.17)$ & $.31(.23)$ \\
\hline \multicolumn{5}{|l|}{ Font Only } \\
\hline Studied hits: & $.75(.16)$ & $.76(.19)$ & $.71(.18)$ & $.66(.21)$ \\
\hline Lure false alarms: & $.25(.15)$ & $.48(.23)$ & $.22(.17)$ & $.43(.17)$ \\
\hline \multicolumn{5}{|l|}{ Novel } \\
\hline Unrelated-matched false alarms: & $.03(.06)$ & $.10(.17)$ & & \\
\hline Critical-Matched false alarms: & $.10(.11)$ & $.10(.19)$ & & \\
\hline
\end{tabular}

